\title{
Disposing of Duplicate Data with Dynamic PoS for Multi User Environment
}

\author{
Nishchitha T S, Dr. K. Thippeswamy \\ Dept. of Computer Science \& Engineering, VTU Regional Centre, Mysuru, Karnataka, India \\ Professor\& H.O.D, Dept. of Computer Science \& Engineering, VTU, Regional Centre, Mysuru, India
}

\begin{abstract}
Dynamic Proof of Storage (PoS) is a profitable custom that empowers a customer to see the respectability of outsourced reports and invigorate the records in a cloud server with an extraordinarily compelling way. Despite the likelihood that a couple of agents have formed unmistakable dynamic PoS in unit customer circumstances, however the inconvenience in multicustomer conditions has not been asked inside and out. A shrewd multi-customer appropriated capacity structure needs the ensured client side cross-customer deduplication framework, that gives a customer to avoid the exchanging strategy and getting the responsibility for records now, once resulting proprietors of a similar archives have exchanged them to the cloud server. To minimal complex of our data, no other present dynamic PoS will support this system. In this paper, we are talented to exhibit the possibility of deduplicatable dynamic check of limit related propose a saving improvement suggested as DeyPoS, to recognize dynamic PoS and secure cross-customer duplication, meanwhile. Considering the troubles of structure contrasts and individual name period, we tend to abuse an exceptional instrument insinuated as Homomorphic Authenticated Tree (HAT). We have a tendency to show the protection of our advancement.
\end{abstract}

Keywords- Deduplication, Proof of ownership, Dynamic proof of storage, Cloud Storage.

\section{INTRODUCTION}

Storage outsourcing is transforming into extra and extra luring to each exchange and instructional exercise as a result of the advantages of low esteem, high openness, and clear sharing. By and large of the capacity outsourcing frames, distributed storage increases wide consideration as of late. A few firms, similar to Amazon, Google, and Microsoft, give their own particular distributed storage administrations, wherever clients will exchange their documents to the servers, get to them from fluctuated gadgets, and offer them with the others. In spite of the fact distributed storage administrations are wide received in current days, there still remain a few security issues and potential dangers.
Data integrity is one among the principal fundamental properties once a client outsources its documents to distributed storage. Clients should be persuaded that the documents keep inside the server don't appear to be altered. Antiquated methods for shielding information uprightness, similar to message confirmation codes (MACs) and advanced marks, require clients to exchange the majority of the records from the cloud server for check that brings about a huge correspondence esteem. These methods don't appear to be suitable for distributed storage benefits wherever clients could check the honesty frequently, similar to every hour.

In this manner, specialists presented Proof of Storage (PoS) for checking the trustworthiness while not downloading documents from the cloud server. Likewise, clients may require numerous dynamic operations, similar to alteration, inclusion, and erasure, to refresh their records, while keeping up the capability of PoS. Dynamic $\mathrm{PoS}$ is anticipated for such powerful operations. In refinement with PoS, dynamic PoS utilize structures, similar to the Merkle tree. Therefore, once unique operations are dead, clients recover labels (which are utilized for respectability checking, similar to MACs and signatures) for the refreshed squares exclusively, as opposed to make for all pieces. to raised see the ensuing substance, we tend to blessing extra insights concerning PoS and dynamic PoS. In these plans, each piece of a document is shared a (cryptographic) tag that is utilized for substantiating the honesty of that square. Once a champion goals to find out the uprightness of a record, it each which way chooses some square lists of the document, and sends them to the cloud server. Steady with these tested files, the cloud server gives back the comparing obstructs adjacent to their labels.

The champion checks the block integrity and index accuracy. The past are regularly specifically reinforced by cryptanalytic labels. An approach to influence the last is that the significant refinement amongst PoS and dynamic PoS. In the greater part of the PoS conspires, the block record is "encoded" into its label, which infers the champion will check the square respectability and file rightness in the meantime. Notwithstanding, dynamic PoS 
can't figure the block records into labels, since the dynamic operations could alteration a few files of non-refreshed hinders, that brings about save calculation and correspondence esteem. For instance, there's a document comprising of one thousand pieces, and a substitution square is embedded behind the second piece of the record. At that point 998 blocks of the primary document are changed, which infers the client ought to create and send 999 labels for this refresh. Structures are acquainted in powerful PoSs with disentangle this test. Accordingly, the labels are snared to the structure rather than the piece files .However, dynamic PoS stays to be enhanced in an exceedingly multi-client climate, on account of the need of cross-client American state duplication on the customer side. This implies clients will skirt the transferring technique and secure the ownership of records now, as long in light of the fact that the transferred documents exist as of now inside the cloud server. This technique will decrease space for putting away for the cloud server, and spare transmission data measure for clients. To the least complex of our information, there are no unique PoS that may bolster secure cross-client duplication.

\section{LITERATURE SURVEY}

1] Title: A Secure and Dynamic Multi-keyword Ranked Search Scheme over Encrypted Cloud Data

Authors: Zhihua Xia, Xingming Sun, Qian Wang.

Description: In this paper, a safe, temperate and dynamic hunt component is anticipated, that backings not exclusively the right multi-catchphrase various leveled seek however conjointly the dynamic erasure and addition of reports. We tend to develop an exceptional watchword adjusted parallel tree in light of the fact that the record, and propose an "Avaricious Depth-first Search" algorithmic program to get higher intensity than direct hunt. Furthermore, the parallel hunt process is controlled to extra scale back the time cost. the well being of the subject is ensured against 2 danger models by abuse the protected kNN algorithmic program. Test comes about show the intensity of our anticipated subject. There is a unit still a few test issues in outspread SE plans. Inside the anticipated topic, proprietor is chargeable for producing change data and causation them to the cloud server.

\section{2] Title: Outsourced proofs of retrievability.}

Authors: F. Armknecht, J.-M. Bohli, G. O.

\section{Karame, Z. Liu, and C. A. Reuter}

Description: In this paper, we characterize the idea of Externalize evidences of retrievability (OPOR), in which clients can assignment an unequivocal inspector to perform also, check POR with the specialist co-op. We contend that the OPOR setting is to security dangers that a current POR security model has not been covered. We propose a formal structure and a security demonstrate for OPOR. We then propose an embodiment of OPOR which expands upon the provably secure private POR plot,we show its security in our suggested security display. We execute a model rely on upon our answer, and survey its execution in a reasonable cloud setting. Our assessment comes about demonstrate that our proposal limits client exertion, incurs avoidable overhead on the examiner and incredibly enhances over current openly certain POR.

\section{3] Title: Security and Privacy in Cloud Computing}

Authors: Minqi Zhou, Rong Zhang, Wei Xie, Weining Qian, Aoying Zhou

Description: Circulated registering transforms into an in vogue expression nowadays. A steadily expanding number of associations wander into Cloud and give benefits above on it. In any case, security and assurance issues drive strong deterrent for customers' assignment of Cloud structures and Cloud organizations. We viewed the security and assurance concerns presented by a measure of Cloud Computing system providers in this paper. Coincidentally, those stresses are not adequate. More prominent security frameworks should be passed on in the Cloud condition to finish the 5 destinations (i.e. availability, order, data uprightness, control and audit) and furthermore security acts should be changed to modify another association among customers and providers in the literature of the cloud.

4] Title: Enabling public verifiability and data dynamics for storage security in cloud computing Authors: Q. Wang, C. Wang, J. Li, K. Ren, and W. Lou Description: Distributed storage has been getting Notoriety since its flexibilty and pay as you go way. Be that as it may, this most recent kind of capacity demonstrate likewise accompanies security challenges. This paper expounds the issue of guaranteeing information uprightness in distributed storage. In the Proof of Retrievability (PoR) demonstrate, after externalize the preprocessed information to the server, the customer will expel its nearby duplicates and just store a less measure of meta information. From that point onward, the customer will inquire the server to give a proof that its information can be downloaded accurately. Nonetheless, numerous current PoR works apply just to static information. The current changing adaptation of PoR plan has a proficiency issue. In this paper, we expound the static PoR plan to evolving situation. Which implies, the customer can perform refresh operation e.g.:

Addition, cancellation and change. After each refresh, the customer can in any case identify the information misfortunes regardless of the possibility that the server tries to conceal them. We make a new form of trusted 
information structure rely on upon a $\mathrm{B}+$ tree and a merkle hash tree. We can call it Cloud Merkle $\mathrm{B}+$ tree. By blending the CMBT with the BLS signature, we suggest a dynamic variant of PoR plan. Comparing with the current dynamic PoR plot, our most pessimistic scenario correspondence many-sided quality is $\mathrm{O}(\log n)$ as opposed to $\mathrm{O}(\mathrm{n})$.

5] Title: Practical dynamic proofs of Retrievability.

Authors: E. Shi, E. Stefanov, and C Papamanthou

Description: We suggest a changing PoR plot with contiguous customer stockpiling whose data transmission cost is moderately equivalent to a Merkle hash tree, in this way being extremely useful. Our development dominates the developments of Stefanov et. al. furthermore, Cash et. al., both speculatively and by and by. In particular, for $n$ number of externalized squares of beta bits every, altering a square requires beta+O data transfer capacity and $\mathrm{O}($ betalog $\mathrm{n})$ server calculation. Overviews are likewise exceptionally effective, requiring beta+O (lambda^2log $n)$ data transfer capacity. We likewise demonstrate to fabricate our conspire freely error free, giving the first changing PoR conspire with such a property. We at last give an extremely proficient execution of our plot.

\section{PROPOSED SYSTEM}

In this System show considers two sorts of substances: the cloud server and clients, for each document, unique client is the client who transferred the record to the cloud server, while ensuing client is the client who demonstrated the responsibility for document yet did not really transfer the record to the cloud server.

There are five stages in a deduplicatable dynamic PoS framework:

1) Pre-process

2) Upload

3) Deduplication

4) Update

5) Proof of storage

In the pre-process stage, clients mean to transfer their neighborhood documents. The cloud server chooses whether these documents ought to be transferred. In the event that the upload procedure is without a doubt, go into the transfer stage; generally, go into the deduplication stag.

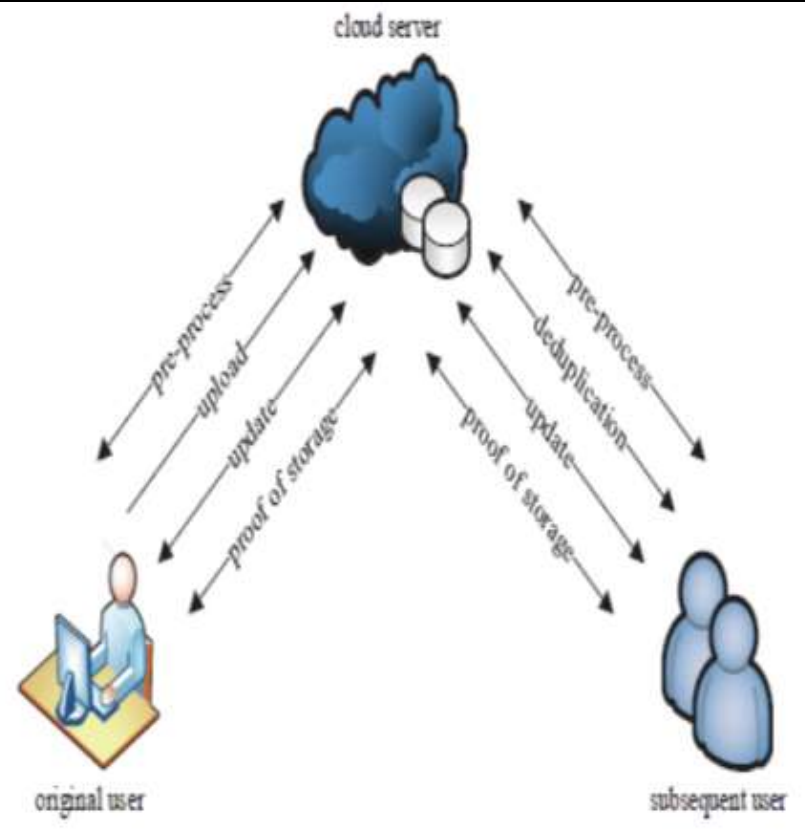

Fig 1: The system model of deduplicatable dynamic PoS

In the upload stage, the documents to be transferred don't exist in the cloud server. The first clients encodes the neighborhood records also, transfer them to the cloud server. In the duplication stage, the documents to be transferred as of now exist in the cloud server. The ensuing clients have the documents locally and the cloud server stores the verified structures of the records. Consequent clients need to persuade the cloud server that they claim the records without transferring them to the cloud server. In the proof of storage phase, users exclusively have a little consistent size data locally and that they have to analyze regardless of whether the records square measure reliably hang on inside the cloud server while not downloading them. The records won't not be transferred by these clients anyway they pass the deduplication part and demonstrate that they require the possessions of the documents. Take note of that, the refresh part and furthermore the evidence of capacity part will be dead numerous circumstances inside the life cycle of a record. Once the ownership is checked, the clients will haphazardly enter the refresh part and furthermore the confirmation of capacity part while not keeping the principal documents locally.

\section{CALCULATION}

\section{1] User Module:-}

- New User

-Understudy or, on the other hand Staff and so forth.

- User login in framework

- Client Upload document in framework.

- User select benefit or characteristic first e.g. understudy or staff 
- Browse Text File to Upload and tap on Upload catch and produces label petition for it.

- If label exist in server database then document is deduplicated and print message

- document as of now exist, then give evidence of proprietorship pointer to this client of existing document for getting to and this client is additionally proprietor of that current document.

- If tag not exists in server database then document is one of a kind then scramble document and put away on cloud organizer in drive.

- User additionally can download document from cloud.

- Client demonstrates all documents that his own transferred i.e. one of a kind document and deduplicated record.

- tap on download connection to download that record

\section{2] Access File}

- Client demonstrates all documents for his quality transferred by proprietor of document.

- tap on download connection to download that document

\section{3] Subsequent User}

-This client are those client who transfer documents on cloud and if record they transfer on cloud is copy or officially existing on cloud then they end up plainly resulting client of document.

-They get responsibility for document and they can get to that record.

\section{CONCLUSION}

We arranged the immense necessities in multi-client cloud capacity frameworks and presented the model of deduplicatable dynamic PoS. we had build up a one of a kind apparatus known as HAT that is Associate in Nursing prudent real structure. Upheld HAT, we had arranged the essential sensible deduplicatable dynamic PoS subject known as DeyPoS and confirm its security inside the arbitrary prophet show. The examination demonstrate that our DeyPoS usage is effective, particularly when the document measure and the quantity of the tested squares are extensive.

\section{ACKNOWLEDGEMENTS}

A sincere thanks to Dr. K . Thippeswamy, Head of the Department of CS \& E, VTU PG Centre Mysuru, Mrs.Shashirekha, Assistant professor, Department of CS \& E, VTU PG Centre mysuru and to my friends.

\section{REFERENCES}

[1] H. Shacham and B. Waters, "Compact Proofs of Retrievability," Journal of Cryptology, vol. 26, no. 3, pp. 442-483, 2013.
[2] Z. Mo, Y. Zhou, and S. Chen, "A dynamic proof of retrievability (PoR) scheme with o(logn) complexity," in Proc. of ICC, pp. 912-916, 2012.

[3] E. Shi, E. Stefanov, and C. Papamanthou, "Practical dynamic proofs of retrievability," in Proc. of CCS, pp. 325-336, 2013.

[4] C. Erway, A. K"upc "u, C. Papamanthou, and R. Tamassia, "Dynamic provable data possession," in Proc. Of CCS, pp. 213-222, 2009.

[5] S. Halevi, D. Harnik, B. Pinkas, and A. ShulmanPeleg, "Proofs of ownership in remote storage systems," in Proc. of CCS, pp. 491-500, 2011.

[6] Z. Ren, L. Wang, Q. Wang, and M. Xu, "Dynamic Proofs of Retrievability for Coded Cloud Storage Systems," IEEE Transactionson Services Computing, vol. PP, no. 99, pp. 1-1, 2015.

[7] R. Tamassia, "Authenticated Data Structures," in Proc. of ESA,pp. 2-5, 2003.

[8] J. Yuan and S. Yu, "Efficient public integrity checking for cloud data sharing with multi-user modification," in Proc. of INFOCOM, pp.

[9] R. Gennaro and D. Wichs, "Fully Homomorphic Message Authenticators," in Proc. of ASIACRYPT, pp. 301-320, 2013.

[10] G. Ateniese, R. Di Pietro, L. V. Mancini, and G. Tsudik, "Scalable and Efficient Provable Data Possession," SecureComm, pp. 1- 10, 2008. homomorphic identification protocols,". 